\title{
On predictive modelling of yield stress increase in fresh cement paste
}

\author{
Haiqin Huang and Andreas Zilian* \\ University of Luxembourg, 6, Avenue de la Fonte, L-4364 Luxembourg
}

Freshly mixed concrete is composed of cement paste as fluid-like component and aggregates as solid particulate component. Development of yield stress in fresh mortar is significant for reliable and high-quality concrete operation as too low or too high yield stress causes process-ability challenges like the multi-layer problem, aggregate flocculation or blockage. Traditional experimental tests on the evolution of yield stress are costly since a wide spectrum of different material and environmental conditions needs to be studied. Progressing hydration, thermal effects and mechanical loading/unloading may influence spatial distribution of the apparent yield stress. However, this local information is very difficult to obtain using traditional laboratory tests and virtual fresh concrete testing is therefore promising.

In the context of a single-component flow approach to homogenized modelling of the cement paste composition at the macro-scale, the microstructural evolution affecting the development and distribution of apparent yield stress is taken into account by a thermo-chemo-mechanical phase-field model bridging the micro-macro length scales by using a Ginzburg-Landautype free energy function. The phase-field, that describes the smooth spatial transition between fluid-like and solid-like behaviour of cement paste in the setting phase, is governed by bond-building chemical processes (hydration) and reversely-acting mechanical effects (bond breakup). The resulting set of coupled nonlinear advection-reaction-diffusion equations are formulated in the Eulerian framework and discretized in space using the weighted residuals method and finite differences in time. Implementation of the predictive model is demonstrated using the FEniCS framework, together with numerical examples supporting model validation.

Copyright line will be provided by the publisher

\section{Introduction}

The development of yield stress of fresh cement paste (FCP) is significant to proper placement of fresh concrete. Changing in the yield stress of cement paste can lead to sedimentation of coarse aggregates in fresh concrete mix. Indeed, sedimentation of the aggregates can be greatly reduced if the yield stress of cement paste is large enough compared to the gravity forces induced by the density difference of the aggregates. In addition, traditional experimental tests (slump test, viscometer test) on the evolution of yield stress are costly since a wide spectrum of different material and environmental conditions is required to be taken into account over time. Therefore, predictive modelling of yield stress evolution of fresh cement paste is of interest.

\section{Mathematical Model}

In the fresh state of concrete, generally in the first couple of hours, FCP is a largely homogeneous mixture of small particles (cement grains) and high content of water. The conservation of mass and momentum of an incompressible flow are considered as follows using the Eulerian description,

$$
\begin{aligned}
& \nabla \cdot \mathbf{v}=0, \\
& \rho \frac{\partial \mathbf{v}}{\partial t}+\rho(\mathbf{v} \cdot \nabla) \mathbf{v}=\nabla \cdot \sigma+\rho \mathbf{b},
\end{aligned}
$$

where $\rho, \mathbf{v}, \mathbf{b}, t$ is density, velocity, specific body force and time, respectively.

During operation of fresh concrete, the mechanical work and viscous dissipation can be neglected when compared to the heat release of hydration in energy balance. We can assume that the mixture is locally in thermal equilibrium. The energy balance equation then regards as

$$
\rho C_{p} \frac{\partial \theta}{\partial t}+\rho C_{p} \mathbf{v} \nabla \theta+\nabla \cdot \mathbf{q}-\dot{Q}=0
$$

where $C_{p}, \theta$ are specific isobaric heat and temperature, respectively. Using Fourier's law, the heat flux is $\mathbf{q}=-k \nabla \theta$ with thermal conductivity $k . \dot{Q}$ is the rate of heat released by chemical reactions described here via the hydration degree as proposed by Gawin et al. [1].

Fresh cement paste is considered as viscoelastic material as its response is that of a medium between an elastic solid and a viscous fluid under external loading. Experimental observation shows viscoelastic behavior with a time retarded strain using creep/recovery measurement. A unified constitutive model for fresh cement paste should be capable to describe elastic,

* Corresponding author: e-mail andreas.zilian@uni.lu 
viscoelastic and plastic properties and their changes over time with progressing cement hydration. The unified constitutive model for fresh cement paste during its fresh state can start from the Bingham model, which shows good agreement for FCP in the dormant stage. While during setting, the transition from a liquid-like material to a solid-like material takes place as a consequence of the CSH products during hydration. To the end of the fresh state, the FCP becomes a porous solid medium. This phenomenological liquid-solid transition can be described by a phase-field variable $\phi$. Therefore, the unified constitutive model used here is a combination of viscoplastic Bingham model and elastoviscoplastic model. The total deviatoric part of Cauchy stress can be described as linear combination of viscoplastic liquid-limit $\mathbf{T}^{v p}$ and elastoviscoplastic solid-limit $\mathbf{T}^{v e}+\mathbf{T}^{v p}$. Then, the total Cauchy stress $\sigma$ is rearranged using a phase-field variable $\phi$ as follows,

$$
\sigma=-p \mathbf{I}+\mathbf{T}^{v p}+\phi \mathbf{T}^{v e}, \quad \mathbf{T}^{v p}=2 \mu \mathbf{D}, \quad \lambda \mathbf{T}^{v e}+\mathbf{T}^{v e}-2 \mu_{\mathrm{m}} \mathbf{D}=\mathbf{0},
$$

where pressure $p$, identity tensor $\mathbf{I}$, relaxation time $\lambda$, rate of deformation tensor $\mathbf{D}$, macro-viscosity $\mu=\mu_{0}+\frac{\tau_{0}}{2(\sqrt{\mathbf{D}: \mathbf{D}}+\varepsilon)}$ and micro-viscosity $\mu_{\mathrm{m}}$. The the upper-convected Maxwell derivative $\stackrel{\nabla}{\mathbf{T}}=\frac{\partial \mathbf{T}}{\partial t}+(\mathbf{v} \cdot \nabla) \mathbf{T}-(\nabla \mathbf{v}) \mathbf{T}-\mathbf{T}(\nabla \mathbf{v})^{\mathrm{T}}$ is objective.

Phase-field models for microstructure evolution are based on the phase transition formalized by Laudau and Ginzburg. The evolution of the transition of cement paste is primarily determined by the percolation threshold of the hydration products. Therefore, the phase-field variable $\phi$ describing liquid-solid transition can be considered as being mainly driven by the heat rate or the rate of hydration degree. Setting $\frac{\partial F}{\partial \phi}=-\beta_{m}\left(\frac{\partial \phi}{\partial t}+\mathbf{v} \cdot \nabla \phi\right)$, the phase-field evolution equation for the model results as follows, governed by the force $g_{\Gamma}$ in the double-well potential $F\left(\phi, g_{\Gamma}\right)$,

$$
\beta_{m}\left(\frac{\partial \phi}{\partial t}+\mathbf{v} \cdot \nabla \phi\right)=\xi^{2} \Delta \phi+\phi(1-\phi)\left(\phi-\frac{1}{2}+g_{\Gamma}\right) .
$$

The driving force $g_{\Gamma}$ was proposed as an arctan function of temperature $\theta$ for crystal growth and steel solidification by Ricken et al. [2]. The main reason for the liquid-solid transition of fresh cement paste is hydration. Therefore the driving force $g_{\Gamma}$ should be a function of rate of hydration degree. Inspired by Ricken et al. [2], the driving force $g_{\Gamma}$ for fresh cement paste can be considered as a function of rate hydration degree, $g_{\Gamma}=\beta_{H} \dot{\Gamma}$. The weighted residuals methods is applied to the strong-form governing equations and the resulting weak form is discretized with the finite element method using the FEniCS framework.

\section{Ring test example}

The FCP is situated between two concentric cylinder with outer radius $r_{o}=0.1 \mathrm{~m}$ and inner radius $r_{i}=0.025 \mathrm{~m}$. The inner cylinder is subject to a constant tangential velocity $v_{i}=2.5 \times 10^{-4} \mathrm{~m} / \mathrm{h}$, while the velocity at the outer is zero. Both inner and outer boundary are adiabatic and initial temperature, hydration degree, phase-field variable and solid volume fraction are given as $\theta_{0}=293.15 K, \Gamma_{0}=0.1, \phi_{0}=0.01$, respectively. In order to compare with the global yield stress and global viscosity tested by the experiments, the global yield stress $\tau_{g}$ and global apparent viscosity $\mu_{g}$ of fresh cement paste can be predicted using our model by considering $\tau_{g}=\tau_{0}+\phi\left\|\mathbf{T}^{\mathrm{ve}}\right\|$ and $\mu_{g}=\frac{\|\mathbf{T}\|}{\|\mathbf{D}\|}$. The evolution of global yield stress in Fig. 2 and global viscosity in Fig. 3 of FCP, slowly increase in the first 3 hours as expected in the experimental tests and predictive model by Petit [3] during the dormant stage. These quantities rapidly increase after 4 hours when entering the setting stage, which indicates the liquid-solid transition.

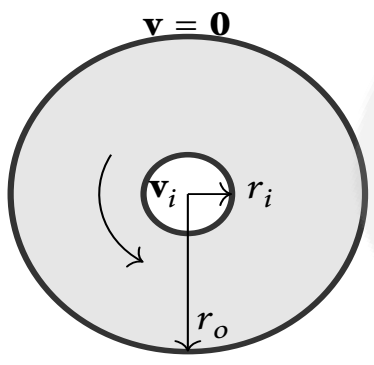

Fig. 1: Problem setup.

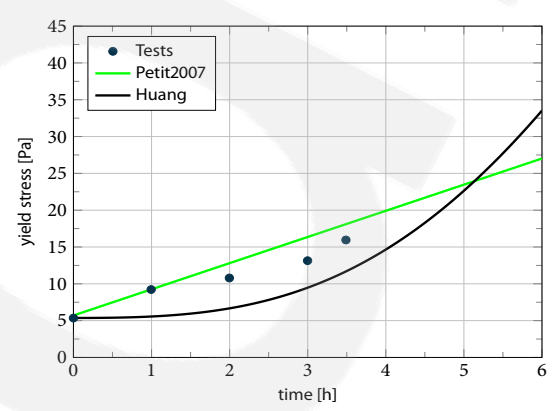

Fig. 2: Apparent yield stress evolution.

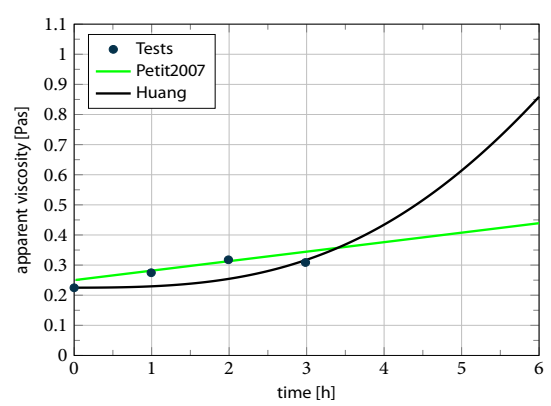

Fig. 3: Apparent viscosity evolution.

\section{References}

[1] D. Gawin, F. Pesavento and B. A. Schrefler, Hygro-thermo-chemo-mechanical modelling of concrete at early ages and beyond. Part I: Hydration and hygro-thermal phenomena, International Journal for Numerical Methods in Engineering, 67, 299-331 (2006).

[2] L Moj, M. Foppe, R. Deike and T. Ricken, Micromacro modelling of steel solidification: A continuum mechanical, biphasic, twoscale model including thermal driven phase transition, GAMM-Mitt, 40, 125-137 (2017).

[3] J. Y. Petit, E. Wirquin, Y. Vanhove and K. Khayat, Yield stress and viscosity equations for mortars and self-consolidating concrete, Cement and Concrete Research, 37, 655-670 (2007). 